\title{
Responsabilidad ética del anestesiólogo ante los cambios climáticos
}

\section{Ethical responsibility of the anesthesiologist in the face of climate change}

Dr. José Manuel Portela-Ortíz,* Dra. Adriana Enríquez-Barajas ${ }^{\ddagger}$

Citar como: Portela-Ortíz JM, Enríquez-Barajas A. Responsabilidad ética del anestesiólogo ante los cambios climáticos. Rev Mex Anestesiol. 2022; 45 (1): 5-6. https://dx.doi.org/10.35366/102894

$\mathrm{D}$ esde la década de 1950 se ha establecido que los cambios climáticos antropogénicos son una realidad. La Organización Mundial de la Salud predice que tales cambios con sus efectos en la contaminación ambiental, el agua potable, la producción de alimentos y los eventos meteorológicos adversos producen ya 250,000 muertes por año ${ }^{(1,2)}$.

Los gases anestésicos son una proporción pequeña de los gases con efecto invernadero; sin embargo, es responsabilidad de los anestesiólogos minimizar el daño por estos gases, además, existe la exposición del personal de salud a estos mismos ${ }^{(3,4)}$.

De los gases anestésicos, el $\mathrm{N}_{2} \mathrm{O}$ y los halogenados que tienen cloruro y bromuro como enfluorano y halotano, además de ser gases de efecto invernadero, depletan la capa de ozono. El efecto invernadero se produce porque estas moléculas absorben e impiden la salida de energía térmica infrarroja aumentando el calentamiento terrestre. El concepto de potencial global de calentamiento toma en cuenta las propiedades radiantes y atmosféricas comparadas con el biotipo del bióxido de carbono $\left(\mathrm{CO}_{2}\right)$, es decir, compara la contribución de los gases de efecto invernadero con la misma masa de $\mathrm{CO}_{2}$ en un período determinado. La Tabla 1 muestra la vida media de los gases anestésicos más utilizados ${ }^{(5)}$.

La capacidad de calentamiento a 100 años (gwp100) ha sido calculada en 893 equivalentes de $\mathrm{CO}_{2} / \mathrm{kg}$ para el desfluorano, comparado con 48 para el sevofluorano y 142 para el isofluorano. Para situarlo en contexto, una hora de anestesia con desfluorano equivale a manejar entre 235 a 475 millas; mientras que con sevofluorano equivale a manejar un auto por 18 millas ${ }^{(5)}$.

Si bien en los países desarrollados las concentraciones en las salas quirúrgicas de gases anestésicos exhalados son monitorizadas para mantenerlas en límites seguros, en los países de ingresos medios y bajos es raro que se determinen estas mediciones, siendo posibles los efectos adversos en la salud de los que están expuestos a $\operatorname{ellos}^{(6)}$. La no utilización de óxido nitroso y minimizar el flujo de gases frescos son recomendaciones avaladas por múltiples organizaciones internacionales, para disminuir este impacto ambiental. Se deberá evitar el uso de $\mathrm{N}_{2} \mathrm{O}$, a menos que se compruebe que reduce la morbimortalidad en los pacientes. Recientemente, han existido avances en la reducción y eliminación de los gases anestésicos de desecho. Esto se ha logrado por la utilización de sistemas de control automático de la administración de los gases anestésicos, la destrucción fotoquímica y el uso de reflectores de gases anestésicos (sistema anaconda) ${ }^{(7-9)}$.

Habiendo visto las consecuencias del uso de agentes inhalados, la anestesia total intravenosa (TIVA) emerge como una candidata para ser tomada en cuenta. El índice PBT analiza el potencial riesgo al medioambiente de diversos agentes farmacéuticos; analiza la persistencia (P) la acumulación (B) y la toxicidad (T). Cada uno de estos valores pueden ser de cero a tres, el índice PBT tiene un rango de cero a nueve y a mayor puntuación mayor riesgo

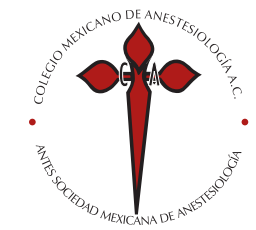

* Anestesiólogo e intensivista. Coordinador del Departamento de Anestesiología Hospital Ángeles Pedregal. Profesor titular de Anestesiología, Universidad La Salle. ¥ Anestesióloga e intensivista. Hospital Ángeles Pedregal.

Correspondencia: Dr. José Manuel Portela-Ortíz Hospital Ángeles Pedregal. Camino a Sta. Teresa 1055- S, Héroes de Padierna, 10700 Ciudad de México, CDMX. Departamento de Enseñanza. E-mail: jmportela55@gmail.com 
Tabla 1: Vida media de los gases anestésicos más utilizados.

\begin{tabular}{|c|c|}
\hline Componente & Vida media \\
\hline $\mathrm{N}_{2} \mathrm{O}$ & 114 \\
\hline CFCs & $50-100$ \\
\hline $\mathrm{CO}_{2}$ & $5-200$ \\
\hline Desflurano & $8.9-21$ \\
\hline Halotano & $1-7$ \\
\hline Isoflurano & $2.6-5.9$ \\
\hline Sevofluorano & $1.1-5.2$ \\
\hline
\end{tabular}

CFCs = clorofluorocarbonados.

ambiental. El propofol tiene un riesgo de cuatro, por lo cual se recomienda que el desecho sea incinerado. Administrar el propofol en viales de $20 \mathrm{~cm}^{3}$ ha demostrado disminuir la cantidad de desecho en el departamento de anestesia. La anestesia del neuroeje o regional tiene los más bajos índices de riesgo ambiental ${ }^{(10-12)}$.

Otras fuentes de contaminación en la anestesiología son los plásticos y los ftalatos, utilizándose este último para lograr la flexibilidad de los productos de PVC. Los ftalatos son tóxicos y su incineración forma dioxinas y fluranos. Los hospitales son una de las principales fuentes de estos tóxicos. Las dioxinas y los fluranos son fuentes bien reconocidas de carcinogénesis, alteraciones endocrinas, afecciones inmunes del sistema reproductivo y del desarrollo neurológico. Por lo tanto, las organizaciones norteamericanas y europeas han dado la señal de alarma y la recomendación de reducir los productos que contengan $\mathrm{PVC}^{(13)}$.

El ciclo de vida del equipo anestésico. Se entiende por ciclo de vida, desde el punto de vista ecológico, al análisis de cada producto utilizado, desde el inicio de obtención de sus materiales, transformación, transporte, posible reutilización y desecho en forma segura, para conocer en forma apropiada su potencial contaminador.

En el clima actual, con énfasis en el control de infecciones, la utilización de equipo desechable se ha incrementado notablemente. Al estudiar el ciclo completo de la vida de cada equipo utilizado en anestesia se pueden hacer decisiones racionales, tanto desde el punto de vista económico, administrativo y ecológico, hasta de control de infecciones. Muchos de los equipos desechables no incluyen en su costo un ciclo total de su huella ecológica, por lo tanto, se llega a la conclusión de que son una buena opción. Existen compañías que se encargan del esterilizado, reempacado y funcionalidad de estos equipos, produciendo ahorros sustanciales en el medioambiente y en costos. En efecto, el ser ecológico conlleva ventajas económicas ${ }^{(6)}$.

Según los datos actuales, quedan a lo sumo dos décadas para impedir el cambio climático, de no lograrlo, enfrentaremos sus efectos magnificados. Por lo anterior, tenemos la responsabilidad y el compromiso con las próximas generaciones para lograrlo.

\section{REFERENCIAS}

1. Pachauri RK, Allen MR, Barros VR, et al. Intergovernmental Panel on Climate Change; Core Writing Team; Climate Change 2014: Syntesis Report. Contribution of Working Groups I, II and III to the Fifth Assessment Report of the Intergovernmental Panel on Climate Change 2015. [Accessed July 23, 2020]. Available in: https://www.ipcc.ch/report/ ar5/ser/.

2. World Health Organization. Climate Change and Health. Fact Sheet 266. Last updated February 1, 2018. [Accessed July 23, 2020]. Available in: www.who.int/mediacentre/factsheets/fs266/en/

3. Boiano JM, Steege AL. Precautionary practices for administering anesthetic gases: a survey of physician anesthesiologists, nurse anesthetists and anesthesiologist assistant. J Occup Environ Hyg. 2016;13:782-793.

4. Teschke K, Abanto Z, Arbour L, et al. Exposure to anesthetic gases and congenital anormalies in offspring of female registered nurses. Am J Ind Med. 2011;54:118-127.

5. Sulbaek AMP, Nielsen OJ, Wallington TJ, Karpichey B, Sander SP. Medical intelligence article: assessing the impact on global climate forma general anesthetic gases. Anesth Analg. 2012;114:1081-1085.

6. Axelrod D, Bell C, Feldman J, et al. Greening the Operating Room and Perioperative Arena: Environmental Sustainability for Anesthesia Practice. American Society of Anesthesiologist. 2014. [Accessed June 2, 2020]. Available in: https://www.asahq.org/about-asa/governance-andcommittees/asa-committees/committee-on-equipment-and-facilities/ environmental-sustainability/greening-the-operating-room
7. Tay S, Weinberg L, Peyton P, Story D, Briedis J. Financial and environmental costs of manual versus automated control of end-tidal gas concentrations. Anaesth Intensive Care. 2013;41:95-101.

8. Epstein RH, Dexter F, Maguire DP, Agarwalla NK, Gratch DM. Economic and environmental considerations during low fresh gas flow volatile agent administration after change to a nonreactive carbon dioxide absorbent. Anesth Analg. 2016;122:996-1006.

9. Rauchenwald V, Rollins MD, Ryan SM, et al. New method of destroying waste anesthetic gases using gas-phase photochemistri. Anesth Analg. 2020;131:288-297.

10. AstraZeneca Pty Ltd. Material Safety Data Sheet: Diprivan (Propofol) 1\% \& 2\%. 2012. [Accessed September 21, 2020]. Available in: https// www.astrazeneca.com.au/content/dam/az-au/Material\%20Safety\%20 Data/Diprivanltsupgtl174ltsupgt.pdf

11. Stockholm City Council. Pharmaceuticals and environment: Propofol. 2020. [Accessed July 23, 2020]. Available in: https://www.janus-info. se/beslutsstod/lakemedelochmiljo/pharmaceutical-sandervironment/ databaseenven/propofol.5.30a7505616a041a09b063eac.html

12. AstraZeneca. Environmental risk assessment data: Propol. 2020. [Accessed September 21, 2020]. Available in: https://www.astrazeneca. com/content/dam/az/our-company/Sustainability/2017/Propofol.pdf

13. Safety Assessment of di-(2-ethylhexyl)-phthalate (DEHP) Released from PVC Medical Devises, Available in: hhtp:/www.fda.gov/cdrh/safety/ dehp.html 